\title{
Mitotic instability of B chromosomes during embryo development in Locusta migratoria
}

\author{
M. C. PARDO, M. D. LOPEZ-LEON, E. VISERAS, J. CABRERO \& J. P. M. CAMACHO* \\ Departamento de Genética, Facultad de Ciencias, Universidad de Granada, 18071-Granada, Spain
}

\begin{abstract}
Mitotic instability of $\mathrm{B}$ chromosomes during embryo development has been studied in the locust Locusta migratoria. Direct cytological observation of B chromosome nondisjunction in embryos has shown that it occurs in 2.7 per cent of anaphase and telophase cells, and that this frequency is not significantly different among embryos of 5-9 days of development. We have defined three indices which have been shown to be very useful to quantifying mitotic instability: $R$, the ratio of embryos showing B chromosome instability, $M$, the median of the distribution of $\mathrm{B}$ chromosome numbers in a sample of embryo cells, which has been shown to be a good estimator of the original number of $\mathrm{B}$ chromosomes present in the zygote, and $M I$, the sum of all deviations (in absolute value) of $\mathrm{B}$ numbers with respect to $M$ in the same embryo. Mitotic instability of $\mathrm{B}$ chromosomes is already apparent in 3-day-old embryos and reaches its maximum value on the fifth day of development. The intensity of mitotic instability, as measured by $M I$, varies significantly during the developmental period analysed but no definite trend was observed.
\end{abstract}

Keywords: accumulation, B chromosomes, embryo development, Locusta migratoria, mitotic instability.

\section{Introduction}

B chromosomes of the locust Locusta migratoria (Orthoptera, Acrididae) are present in most natural populations from Europe, Asia, Africa and Australia. The reasons for such a widespread polymorphism are probably the high flight capability (they still swarm in some places in Africa), high female fecundity (they lay an egg pod once a week containing 54 eggs on average) and rapid embryo (about 23 days) and nymphal ( 30 days) developments (Viseras, 1986). Besides the favourable properties of this locust, B chromosomes show a premeiotic accumulation mechanism during development in the male germ line based on their mitotic instability (Nur, 1969; Kayano, 1971; Viseras et al., 1990). Viseras et al. (1990) demonstrated that mitotic instability of B chromosomes is already manifested in 6-day-old embryos, irrespective of sex, and that they are accumulated in the male but not in the female germ line. However, there still remain a number of questions to be solved; for instance, at what time of embryo development does B mitotic instability begin, how does the rate of $\mathrm{B}$ nondisjunction change during

*Correspondence. ontogeny and does it depend on factors like sex or developmental stage? The answers to these questions were the main objectives of the present investigation.

\section{Materials and methods}

Adult males and females of $L$. migratoria were caught in several localities near Granada. After isolating each female for 1 month, those that had not laid fertilized eggs were placed with a male in laboratory cages to obtain, separately, the egg pods laid by each female. A total of seven females yielded enough embryo offspring to perform an analysis of B mitotic instability during embryo development. We will consider all progeny yielded by the same female as a family. For embryo analysis, egg pods were incubated at $28^{\circ} \mathrm{C}$ for $3-13$ days. About half of the eggs in each egg pod were dissected after a certain number of incubation days and the other half were dissected 2 days later.

Embryos older than 5 days were fixed in 1:3 acetic acid:ethanol, and cytological preparations were made as described in Camacho et al. (1991). Three-day-old and 5-day-old embryos, however, were dissected in a drop of the fixation mixture on a slide and after a few seconds were squashed in 50 per cent acetic acid. 
Coverslips were removed after immersion into liquid nitrogen. All preparations underwent the C-banding procedure described in Camacho et al. (1984).

Because mitotic instability of B chromosomes produces variation in $\mathrm{B}$ number among the cells of the same individual, it is necessary to analyse a sample of cells from each individual to obtain an accurate estimation of B number. About 20 cells, wherever it was possible, were analysed from each embryo. However, this number was difficult to achieve in 3-day-old embryos and, for this reason, we studied fewer cells in this type of embryo. Embryos with less than five cells analysed have not been considered for statistical analysis.

\section{Definitions of variables}

Three variables have been used for the quantification of intraindividual $\mathrm{B}$ chromosome variation from each family: (i) $R$ : ratio of embryos showing $\mathrm{B}$ chromosome instability; (ii) $M$ : number of B chromosomes in each individual, represented by the median of the distribution of B numbers in a sample of its cells; and (iii) $M I$ : mitotic instability index, which measures the deviations in B numbers with respect to the median.

The two latter variables will be used to separate the effects of both inheritance and mitotic instability in intraindividual $\mathrm{B}$ chromosome variation. Thus, $M$ may be taken to represent the original $\mathrm{B}$ number in the zygote state, and $M I$ to be a measure of the intensity of mitotic instability. The first assumption is based on the following arguments. Although a young embryo usually shows B chromosome variation, it is certain that when it was a zygote it possessed an integer number of Bs $(0$, $1,2, \ldots)$. While the mean number of Bs observed in an embryo cannot coincide with the zygotic original number of Bs, because it is not an integer number, and as B chromosome distributions are very grouped around central values (see Table 2 in Viseras et al., 1990 ), it is reasonable to think that the original number of $\mathrm{Bs}$ in the zygote could coincide with the median or the mode of the distribution of B numbers in a sample of embryo cells. We have preferred the median for this purpose because it is the more robust central parameter of the distribution although, in fact, median and mode did coincide in the great majority of embryos analysed.

Given that the median represents the number of Bs which a zygote has inherited from its parents, the extent to which this number is modified through development will serve to quantify mitotic instability of the $\mathrm{B}$ chromosomes. For this purpose, we propose that the $M I$ index be calculated as the sum of the absolute values of every deviation in B number with respect to the median, and normalized by dividing by the median and the number of cells analysed so that the index is independent of the number of Bs and the sample size, i.e.:

$M I=\frac{\Sigma\left(\left|M-n_{i}\right| f_{i}\right)}{M \cdot N}$,

where $M$ is the median, $n_{i}$ is the number of $\mathrm{B}$ chromosomes in the different types of cell that do not coincide with $M, f_{i}$ is the number of cells of each particular type and $N$ is the total number of cells analysed.

$R$ comparisons were made by $\chi^{2}$ tests, but $M$ and $M I$ comparisons were performed using the nonparametric Kruskal-Wallis test and Spearman rank correlation because these variables did not fit normal distributions. Analyses were made using programs 3D, $3 \mathrm{~S}$ and $4 \mathrm{~F}$ of the BMDP statistical software (Dixon, 1990).

\section{Results}

\section{Mitotic nondisjunction of $B$ chromosomes}

The cytological nature of standard and B chromosomes of $\boldsymbol{L}$. migratoria has been analysed recently by means of several chromosome banding techniques (Camacho et al., 1991). These studies have revealed that $\mathrm{C}$-banding is the best method for identifying $\mathrm{B}$ chromosomes as they possess the largest $\mathrm{C}$-band in the karyotype, which permits distinguishing and counting the number of Bs in every type of cell, including interphases (Viseras et al., 1990). On this basis, we analysed 263 mitotic anaphase and telophase cells in embryos of $5,6,7,8$ and 9 days from family 1 , in order to observe directly the mitotic nondisjunction (Fig. 1) which is supposed to be the basis of premeiotic accumulation of B chromosomes in L. migratoria. To minimize misinterpretations caused by simultaneous nondisjunction of two Bs to opposite poles, that could be erroneously considered as regular disjunctions, only cells with $1 \mathrm{~B}$ were considered (Table 1). A nonparametric Kruskal-Wallis test showed that the frequency of B nondisjunction is independent of embryo age $\left(H_{4}=2.03, P=0.73\right)$. Thus, the mean rate of nondisjunction was 2.7 per cent in the observed developmental period.

\section{Intraindividual variation in the number of $B$ chromosomes during embryo development}

Our previous studies (Cabrero et al., 1984; Viseras et al., 1990; Camacho et al., 1991) have demonstrated that the number of $\mathrm{B}$ chromosomes varies among the 

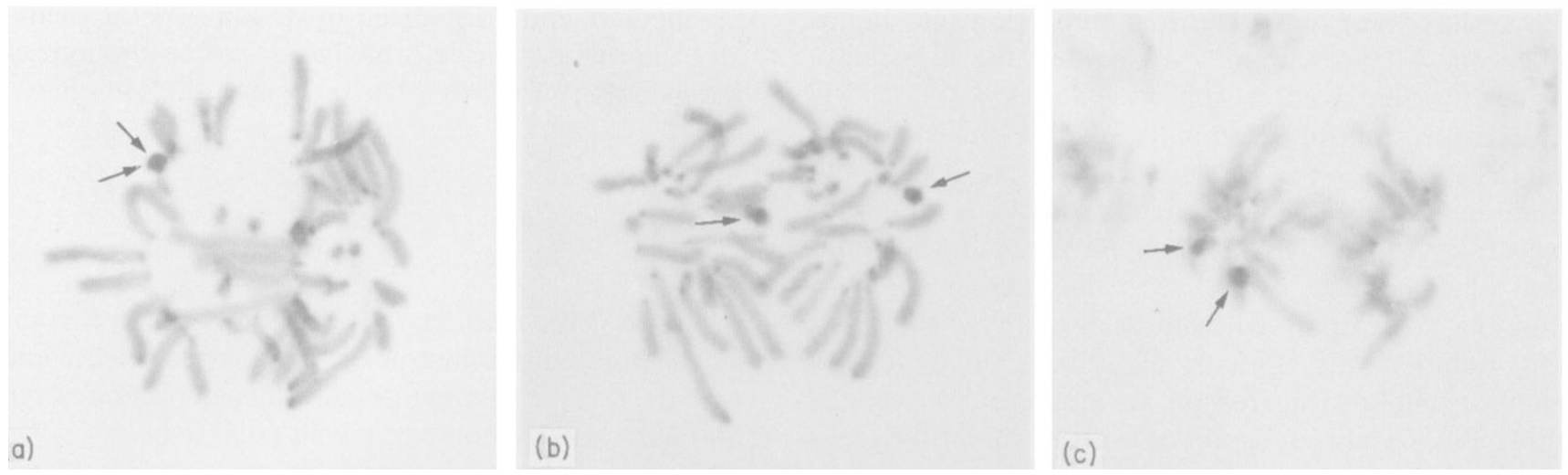

Fig. 1 Embryonic mitotic anaphase cells with a single B chromosome showing (a) the whole B chromosome in a pole with both chromatids still joined (1/0), (b) regular disjunction of the B chromosome (1/1), and (c) both separated chromatids in the same pole $(2 / 0)$. Arrowheads indicate B chromatids.

Table 1 Results of a score of anaphase and telophase cells with a single B chromosome, performed on 5-9-day-old embryos from family 1 . Three types of cells were observed: (i) cells showing the whole $\mathrm{B}$ chromosome in a pole with both chromatids still joined $(1 / 0)$; (ii) cells showing regular disjunction of the B chromosome (1/1); and (iii) cells showing both separated chromatids in the same pole $(2 / 0)$

\begin{tabular}{ccrcr}
\hline Days & $1 / 0$ & $1 / 1$ & $2 / 0$ & Total \\
\hline 5 & 0 & 61 & 2 & 63 \\
6 & 0 & 55 & 2 & 57 \\
7 & 1 & 59 & 0 & 60 \\
8 & 2 & 43 & 0 & 45 \\
9 & 0 & 38 & 0 & 38 \\
Total & 3 & 256 & 4 & 263 \\
\hline
\end{tabular}

cells of a given individual (embryos, adult males and adult females). In the seven families analysed in the present paper, 89.1 per cent of 339 male embryos and 88.7 per cent of 344 female embryos showed B instability, so that $R$ is not dependent on sex $\left(\chi_{1}^{2}=0.031, P=0.86\right)$. Likewise, $M$ and $M I$ are also independent of sex, using the Kruskal-Wallis test (Table 2).

Figure 2a shows the $R$ values observed in families $1-7$, as a whole, with respect to embryo age. The highest change in $R$ was observed between 3- and 5-dayold embryos. A contingency $\chi^{2}$ test showed significant differences among the different age groups $\left(\chi_{7}^{2}=15.79\right.$, $P=0.027)$. However, if we do not consider 3-day-old embryos, then the differences are not significant $\left(\chi_{6}^{2}=1.50, P=0.96\right)$.

B frequency measured by $M$ was not significantly different among embryos of different ages in most families (Fig. 2b). The only exception was family 7 in which a tendency towards an increase of $M$ with age was slightly significant $\left(H_{2}=6.05, P=0.049\right)$. These results suggest that $M$ is resistant to the changes in $\mathrm{B}$ number induced by mitotic instability, during the period of embryo development analysed, as would be expected for a good estimator of the original number of Bs in the zygote.

The mitotic instability index $(M I)$ was variable among embryos of different ages, and the differences were significant in four families (Table 3 ). This suggests the existence of variable intensities of B mitotic instability during embryo development. Spearman rank correlation analyses applied to these four families showed no clear tendency towards an increase or a decrease in $M I$ with respect to embryo age in family 1 $\left(r_{\mathrm{s}}=0.14, P=0.11\right)$, but a clear tendency towards a decrease in family $4\left(r_{\mathrm{s}}=-0.20, P=0.002\right)$ and towards an increase in families $5 \quad\left(r_{s}=0.28\right.$, $P=0.0006)$ and $6\left(r_{\mathrm{s}}=0.36, P=0.0018\right)$.

\section{Discussion}

The accumulation mechanism of B chromosomes in $L$. migratoria is based on preferential nondisjunction of Bs to the germ line during development (Nur, 1969; Kayano, 1971), and it seems to work exclusively in males (Viseras et al., 1990). While nondisjunction is already manifest in 3-day-old embryos, the preferential mechanism, however, can not have operated yet because there is a proportion of embryos showing B mitotic stability that, after development, will actually show B chromosome variation in the germ line, which is the rule for every adult. This must be true unless polar cells do not multiply in 3-13-day-old embryos, in which case B chromosome variation in the germ line would not be detected despite its existence. Bearing 
Table 2 Comparison of the median number $(M)$ and the mitotic instability index $(M I)$ of $\mathrm{B}$ chromosomes between male and female embryos from the same family, by means of the nonparametric Kruskal-Wallis test $(H)$

\begin{tabular}{|c|c|c|c|c|c|c|c|c|c|c|c|}
\hline \multirow[b]{2}{*}{ Family } & \multirow[b]{2}{*}{ Sex } & \multicolumn{5}{|c|}{$M$} & \multicolumn{5}{|c|}{$M I$} \\
\hline & & $N$ & Median & $H$ & d.f. & $P$ & $N$ & Median & $H$ & d.f. & $P$ \\
\hline \multirow[t]{2}{*}{1} & M & 66 & 2.000 & 0.55 & 1 & 0.4582 & 63 & 0.1000 & 0.01 & 1 & 0.9119 \\
\hline & $\mathrm{F}$ & 68 & 2.000 & & & & 68 & 0.1111 & & & \\
\hline \multirow[t]{2}{*}{2} & $\mathbf{M}$ & 16 & 1.000 & 0.25 & 1 & 0.6193 & 13 & 0.0667 & 2.53 & 1 & 0.1120 \\
\hline & $\mathrm{F}$ & 34 & 2.000 & & & & 25 & 0.1579 & & & \\
\hline \multirow[t]{2}{*}{3} & $\mathbf{M}$ & 28 & 1.000 & 0.55 & 1 & 0.4575 & 25 & 0.1750 & 0.14 & 1 & 0.7094 \\
\hline & $\mathrm{F}$ & 28 & 1.500 & & & & 23 & 0.2000 & & & \\
\hline \multirow[t]{2}{*}{4} & $\mathrm{M}$ & 124 & 2.000 & 1.07 & 1 & 0.3016 & 122 & 0.1118 & 1.01 & 1 & 0.3141 \\
\hline & $\mathrm{F}$ & 118 & 2.000 & & & & 116 & 0.1167 & & & \\
\hline \multirow[t]{2}{*}{5} & $\mathbf{M}$ & 70 & 1.000 & 1.08 & 1 & 0.2993 & 62 & 0.0955 & 1.42 & 1 & 0.2332 \\
\hline & $\mathrm{F}$ & 75 & 1.000 & & & & 61 & 0.1364 & & & \\
\hline \multirow[t]{2}{*}{6} & M & 30 & 2.000 & 0.84 & 1 & 0.3604 & 28 & 0.1354 & $<0.01$ & 1 & 0.9533 \\
\hline & $\mathrm{F}$ & 40 & 1.000 & & & & 38 & 0.1423 & & & \\
\hline \multirow[t]{2}{*}{7} & $\mathbf{M}$ & 33 & 2.000 & 6.95 & 1 & 0.0084 & 27 & 0.1250 & 1.71 & 1 & 0.1915 \\
\hline & $\mathrm{F}$ & 22 & 1.000 & & & & 15 & 0.1000 & & & \\
\hline
\end{tabular}

$N$ : number of embryos.

also in mind that mitotic B instability leads to accumulation in males but not in females (Viseras et al. 1990) and that at the embryonic stages there is no difference between the sexes for the incidence and amount of $\mathrm{B}$ mitotic instability, it follows that mitotic instability per $s e$ is not enough to explain B accumulation. On this basis, $\mathrm{B}$ accumulation seems to occur in two welldifferentiated stages: (i) the generation of intraindividual $\mathrm{B}$ chromosome variation during embryo development, that is similar for both sexes; and (ii) the preferential fate of cells with a high number of $B$ chromosomes to the germ line, that operates only in males. Our present results show clear evidence of the first stage. The direct study of the second one is rather difficult because it most probably works during developmental stages that are not amenable to analysis.

It is interesting to comment on some aspects of the mechanism of nondisjunction of B chromosomes. The conservation of chromosome number in eukaryotic organisms during development depends on the accuracy of chromosome transmission during mitosis. A high number of mutations affecting chromosome transmission fidelity in mitosis have been isolated and are currently being studied (see Spencer et al., 1990, for review). One of the most important processes in achieving this fidelity is the formation of the mitotic spindle by microtubule organizing centres. Recent studies of genes involved in the distributive segregation system in Drosophila (Endow et al., 1990; McDonald \& Goldstein, 1990; Zhang et al., 1990) have shown that these loci encode members of the kinesin heavy chain superfamily, so that chromosome movement in the spindle seems to involve the same kind of motors that move the organelles along microtubules in the cytoplasm (Carpenter, 1991). Recent approaches involving studying the movements of microtubules on the kinetochores of isolated chromosomes by video microscopy (Hyman \& Mitchison, 1991) will presumably throw much light on our understanding of chromosome movement during mitosis.

Proteins named INCENPs (inner centromere proteins) have been found between sister chromatids of metaphase chromosomes, and could be involved in chromatid disjunction in anaphase because at the end of metaphase they leave their place in the chromosome and set up transverse lines to the plate in association with polar microtubules outside the kinetochore (Cooke et al., 1987). There are other proteins that also associate with the chromosomes during prometaphase and metaphase and then separate from them to associate with the midzone of the spindle during anaphase (Kingwell et al., 1987; Pankov et al., 1990; Compton et al., 1991; Yen et al., 1991). This makes them candidates for playing a role, like the INCENPs, in the disjunction of sister chromatids. Furthermore, some of them may function prior to anaphase. For instance, CHO1 (an antigen identified using a monoclonal antibody elicited by immunization with spindles isolated from chinese hamster ovary cells) is required for the correct alignment of chromosomes in the metaphase plate (Nislow et al., 1990). Earnshaw \& Bernat (1991) have proposed a double function for these 'chromo- 
(a)

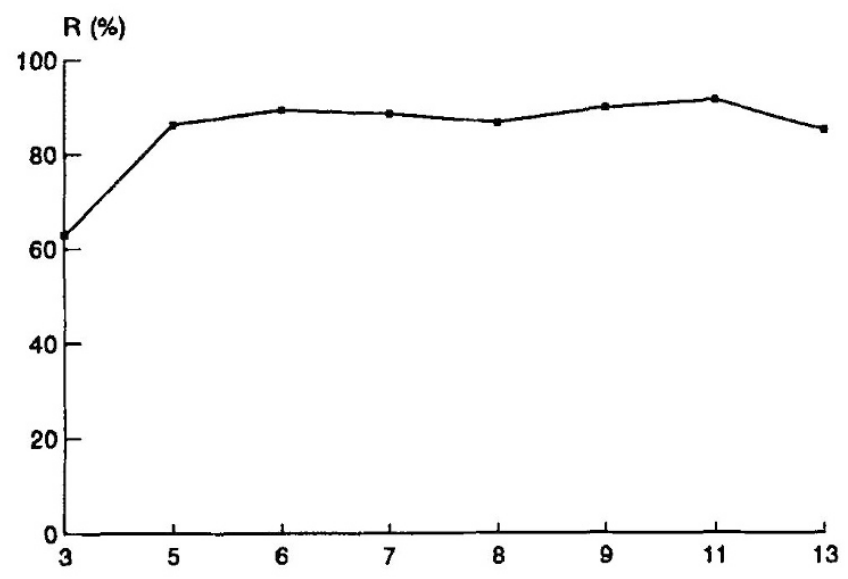

(b)

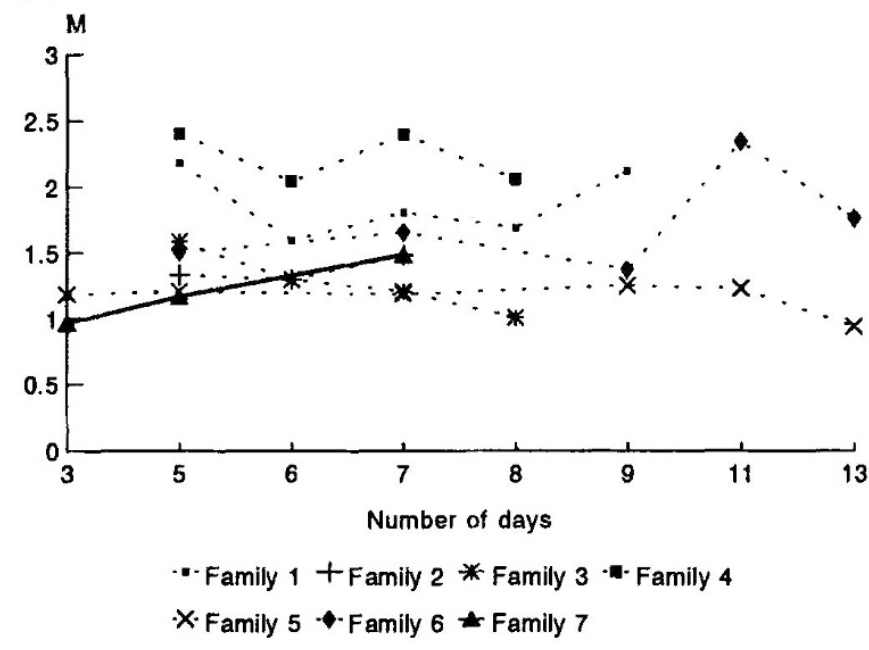

Fig. 2 Variation of $R(\mathrm{a})$ and $M(\mathrm{~b})$ with respect to the number of days of embryo development. Observe in (a) that $R$ increased significantly between 3 and 5 days of incubation and afterwards it was almost uniformly constant. Note in (b) that $M$ was independent of embryo age in six (dotted lines) of the seven families analysed.

some passengers', as they call them, namely a function at the level of metaphase chromosomes (setting them appropriately in the plate, facilitating sister chromatid disjunction, etc.), and another function, in anaphase, acting as cytoskeletal proteins while the mitotic spindle is working.

Nondisjunction of $\mathrm{B}$ chromosomes in L. migratoria could be based on a deficient functioning of proteins involved in chromosome movement, at the level of kinetochore movement or sister chromatid disjunction, so that the sister chromatids of $\mathrm{B}$ chromosomes are inappropriately separated. Future work should be focused on this possibility in order to understand all
Table 3 Comparison of the B mitotic instability index $(M I)$ among embryos of different ages from the same family by means of the nonparametric Kruskal-Wallis test $(H)$

\begin{tabular}{|c|c|c|c|c|c|c|}
\hline \multirow[b]{2}{*}{ Family } & \multirow{2}{*}{$\begin{array}{c}\text { Embryo age } \\
\text { (days) }\end{array}$} & \multicolumn{2}{|c|}{$M I$} & \multirow[b]{2}{*}{$H$} & \multirow[b]{2}{*}{ d.f. } & \multirow[b]{2}{*}{$P$} \\
\hline & & Median & $N$ & & & \\
\hline 1 & $\begin{array}{l}5 \\
6 \\
7 \\
8 \\
9\end{array}$ & $\begin{array}{l}0.1304 \\
0.0917 \\
0.0897 \\
0.0870 \\
0.1739\end{array}$ & $\begin{array}{l}17 \\
21 \\
45 \\
21 \\
27\end{array}$ & 17.21 & 4 & 0.0018 \\
\hline 2 & $\begin{array}{l}5 \\
6 \\
7\end{array}$ & $\begin{array}{l}0.0969 \\
0.1125 \\
0.1750\end{array}$ & $\begin{array}{l}12 \\
16 \\
11\end{array}$ & 2.29 & 2 & 0.3177 \\
\hline 3 & $\begin{array}{l}5 \\
6 \\
7 \\
8\end{array}$ & $\begin{array}{l}0.1625 \\
0.1399 \\
0.1875 \\
0.2440\end{array}$ & $\begin{array}{r}18 \\
6 \\
20 \\
4\end{array}$ & 1.46 & 3 & 0.6921 \\
\hline 4 & $\begin{array}{l}5 \\
6 \\
7 \\
8\end{array}$ & $\begin{array}{l}0.1270 \\
0.0567 \\
0.1146 \\
0.0833\end{array}$ & $\begin{array}{r}95 \\
22 \\
104 \\
19\end{array}$ & 25.67 & 3 & 0.0001 \\
\hline 5 & $\begin{array}{r}3 \\
5 \\
7 \\
9 \\
11 \\
13\end{array}$ & $\begin{array}{l}0.0476 \\
0.0556 \\
0.1429 \\
0.0500 \\
0.2250 \\
0.1500\end{array}$ & $\begin{array}{r}10 \\
22 \\
49 \\
28 \\
29 \\
9\end{array}$ & 26.12 & 5 & 0.0001 \\
\hline 6 & $\begin{array}{r}5 \\
7 \\
9 \\
11 \\
13\end{array}$ & $\begin{array}{l}0.1136 \\
0.0750 \\
0.1750 \\
0.2000 \\
0.2083\end{array}$ & $\begin{array}{r}5 \\
20 \\
39 \\
5 \\
4\end{array}$ & 11.85 & 4 & 0.0185 \\
\hline 7 & $\begin{array}{l}3 \\
5 \\
7\end{array}$ & $\begin{array}{l}0.0909 \\
0.0646 \\
0.1000\end{array}$ & $\begin{array}{l}17 \\
26 \\
27\end{array}$ & 1.49 & 2 & 0.4749 \\
\hline
\end{tabular}

d.f.: degrees of freedom; $P$ : probability.

the details of the accumulation mechanism of $\mathrm{B}$ chromosomes in L. migratoria.

\section{Acknowledgements}

We are indebted to Dr A. González Carmona for his help with statistical procedures and Mrs J. Stocco for her English corrections. This study was partially supported by grants from the Dirección General de Investigación Científica y Técnica (no. PB90-0865) and the Plan Andaluz de Investigación, Grupo no. 3122 (Spain). 


\section{References}

CABRERO, J., VISERAS, E. AND CAMACHO, J. P. M. 1984. The Bchromosomes of Locusta migratoria. I. Detection of negative correlation between mean chiasma frequency and the rate of accumulation of the B's; a reanalysis of the available data about the transmission of these B chromosomes. Genetica, 64, 155-164.

CAMACHO, J. P. M., CABRERO, J., VISERAS, E., LOPEZ-LEON, M. D., NAVAS-CASTILlO, J. AND ALCHE, J. D. 1991. G-banding in two species of grasshoppers and its relationship to $\mathrm{C}, \mathrm{N}$ and fluoroscence banding techniques. Genome, 34, 638-643.

CAMACHO, J. P. M., VISERAS, E., NAVAS, J. AND CABRERO, J. 1984. C-heterochromatin content of supernumerary chromosome segments of grasshoppers: detection of an euchromatic extra segment. Heredity, 53, 167-175.

CARPENTER, A. T. C. 1991. Distributive segregation: motors in the polar wind? Cell, 64, 885-890.

COMPTON, D. A., YEN, T. J. AND CLEVELAND, D. W. 1991. Identification of novel centromere kinetochore associated proteins using monoclonal antibodies generated against human mitotic chromosome scaffolds. J. Cell. Biol., 112, 1083-1097.

COOKE, C. A., HECK, M. M. S. AND EARNSHAW, W. C. 1987. The INCENP antigens: movement from the inner centromere to the midbody during mitosis. J. Cell. Biol., 105, 2053-2067.

DIXON, W. J. (ED.). 1990. BMDP Statistical Software Manual. University of California Press, Berkeley.

EARNSHAW, W. C. AND BERNAT, R. L. 1991. Chromosomal passengers: toward an integrated view of mitosis. Chromosoma, 100, 139-146.

ENDOW, S. A., HENIKOFF, S. AND SOLER-NIEDZIELA, L. 1990 Mediation of meiotic and early mitotic chromosome segregation in Drosophila by a protein related to kinesin. Nature, 345, 81-83.

HYMAN, A. A. AND MITCHINSON, T. J. 1991. Two different microtubule-based motor activities with opposite polarities in kinetochores. Nature, 351, 206-211.
KAYANO, H. 1971. Accumulation of B chromosomes in the germ line of Locusta migratoria. Heredity, 27, 119-123.

KINGWELL, B., FRITZLER, M. J., DECOTEAU, J. AND RATTNER, J. B. 1987. Identification and characterization of a protein associated with the stembody using autoimmune sera from patients with systemic sclerosis. Cell. Motil. Cytoskel., 8, 360-367.

McDONALD, H. B. AND GOLDSTEIN, L. S. B. 1990. Identification and characterization of a gene encoding a kinesin-like protein in Drosophila. Cell, 61, 991-1000.

NISLOW, C., SELLITO, C., KURIYAMA, R. AND McINTOSH, J.R. 1990. A monoclonal antibody to a mitotic microtubule-associated protein blocks mitotic progression. J. Cell Biol., 111, 511-522.

NUR, U. 1969. Mitotic instability leading to an accumulation of B-chromosomes in grasshoppers. Chromosoma, 27, 1-19. PANKOV, R., LEMIEUX, M. AND HANCOCK, R, 1990. An antigen located in the kinetochore region in metaphase and on polar microtubule ends in the midbody region in anaphase, characterized using a monoclonal antibody. Chromosoma, 99, 95-101.

SPENCER, F., GERRING, S. L., CONNELLY, C. AND HIETER, P. 1990. Mitotic chromosome transmission fidelity mutants in Saccharomyces cerevisiae. Genetics, 124, 237-249.

viseras, E. 1986. Análisis de la Naturaleza, Efectos y Transmisión de los Cromosomas Accesorios de Locusta migratoria. Ph.D. Thesis, Universidad de Granada.

VISERAS, E., CAMACHO, J. P. M., CANO, M. I. AND SANTOS, J. L. 1990. Relationship between mitotic instability and accumulation of B chromosomes in males and females of Locusta migratoria. Genome, 33, 23-29.

YEN, T. J., COMPTON, D. A., WISE, D., ZINKOWSKI, R. P., BRINKLEY, B. R., EARNSHAW, w. C. AND CLEVELAND, D. W. 1991. CENP-E, a novel centromere associated protein required for progression from metaphase to anaphase. EMBOJ., 10, 1245-1254.

ZHANG, P., KNOWLES, B. A., GOLDSTEIN, L. S. B. AND HAWLEY, R. S. 1990. A kinesin-like protein required for distributive chromosome segregation in Drosophila. Cell, 63, 1053-1062. 